\title{
Frontières
}

\section{La mort, entre jeux d'ombre et de lumière}

\section{Jean-Jacques Lavoie}

Volume 14, numéro 2, printemps 2002

La mort prononcée

URI : https://id.erudit.org/iderudit/1073962ar

DOI : https://doi.org/10.7202/1073962ar

Aller au sommaire du numéro

Éditeur(s)

Université du Québec à Montréal

ISSN

1180-3479 (imprimé)

1916-0976 (numérique)

Découvrir la revue

Citer ce document

Lavoie, J.-J. (2002). La mort, entre jeux d'ombre et de lumière. Frontières, 14(2),

5-7. https://doi.org/10.7202/1073962ar d'utilisation que vous pouvez consulter en ligne.

https://apropos.erudit.org/fr/usagers/politique-dutilisation/ 


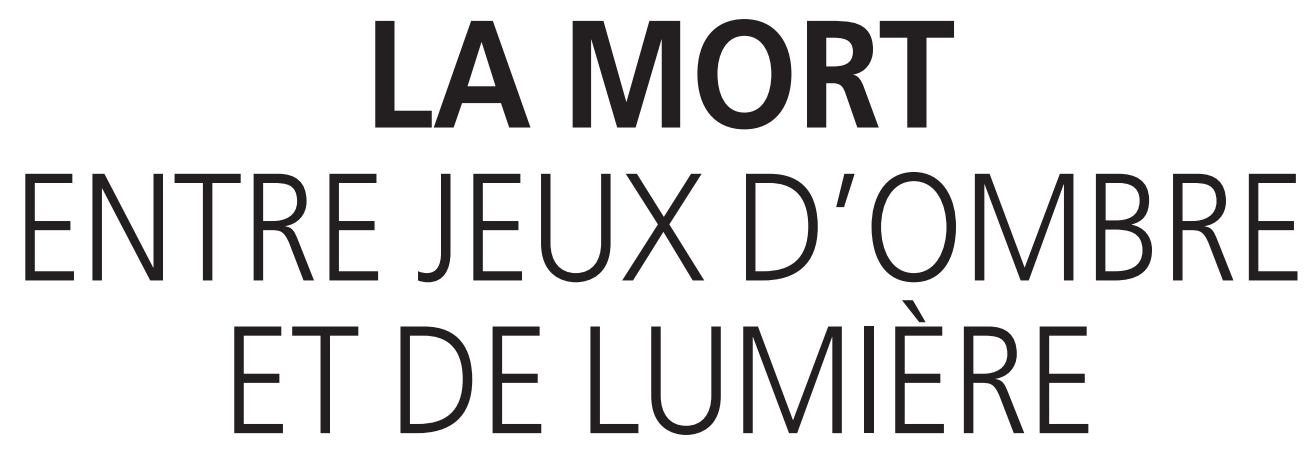

« INTERROGE-T-ON LA MORT ? - ON N'INTERROGE QU'ELLE. »

(JABÈS, 1984, p. 190)

\footnotetext{
Jean-Jacques Lavoie, Ph.D., directeur de la revue Frontières, UQÀM.
}

La mort prononcée : annonce et commémoration. Ce bout de phrase, simple en apparence, fait référence à une réalité d'une grande complexité par son exigence et son urgence. Dans nos sociétés thanatophobes et pourtant mortifères, la mort est prononcée de mille et une façons : on l'annonce à la télévision, qui balance quelques cadavres entre deux publicités, dans les journaux et à la radio, qui décrivent sans cesse de nouveaux carnages, au point qu'on en vient à se demander si la planète ne serait pas devenue un vaste camp d'extermination... ou on fait mémoire sur la place publique avec une plaque ou une sculpture qui commémore un personnage du passé et aussi sur toutes ces affiches de cinéma et de théâtre qui publicisent des tragédies anciennes et modernes et montrent des événements à travers lesquels la mort entre en scène sous le feu des projecteurs.

Le thème de la mort prononcée peut paraître élémentaire puisqu'il se retrouve presque partout sous divers éclairages. Les lecteurs de Frontières sont invités ici à circuler dans des espaces qu'ils connaissent peut-être trop bien, ceux où la parole ouvre une blessure ou ravive une cicatrice en même temps qu'elle éclaire.

\section{PENSER L'IMPENSABLE}

De la mort elle-même ni la philosophie, ni les cartes du tarot, ni la littérature ne peut rien dire, car de la mort il n'existe nulle expérience. Toutefois, comme l'affirme ici en ouverture Michel Clément, on peut parler du mourir et de la conscience de la mort. On peut même dire, à la suite de Sénèque, que c'est pendant la vie entière qu'il faut apprendre à mourir. La mort n'est donc pas qu'événementielle. Elle est une dimension constitutive de l'existence : vivre n'est jamais que vivre sa mort. Comme les philosophes, les concepteurs qui ont proposé un dessin pour la carte de la mort dans le tarot ont été condamnés, pour reprendre une expression de Michel Clément, «à extraire du sens à partir de l'inconnaissable », à produire du discours à partir de dispositifs lacunaires. C'est ce que met en lumière l'article de Danièle Bourque, qui scrute le dit et le non-dit de la mort dans trois versions de cette carte de tarot.

Mourir étant aussi un mot et les mots étant «l'étoffe dont nous sommes faits» (Paz, 1982, p. 37), nous avons donné une place importante à l'analyse de quelques œuvres littéraires qui sont de véritables miroirs de la vie de leurs auteurs, voire de notre condition humaine. Ainsi, les textes d'Hervé Guibert, Hubert Aquin (par Catherine Mavrikakis), Walter Benjamin (par Angela Cozea) - trois auteurs qui ont en commun de s'être suicidés -, Yasmine Chami-Kettani (par Isabelle Larrivée), Jacques Derrida (par Martine Delvaux), les Tragiques grecs et Maurice Maeterlinck (par Yves Jubinville), sont autant de prodigieux tremplin pour interroger les versants nocturnes de la mort qui se dit avec violence ou dans une avance lente et progressive.

\section{MOURIR TOUTE SA MORT}

Entre la mort et soi, il y a la puissance de la médecine. Les stoïciens, nous rappelle Michel Clément, prônaient l'indifférence devant la mort, parce qu'ils la voulaient libre de toute passion. Les médecins sont probablement nombreux à rêver que leurs patients, à qui ils ont une terrible annonce à faire, soient de fidèles adeptes des philosophes du Portique. Comme les partisans du stoïcisme sont de nos jours fort peu nombreux, on exigera des médecins qu'ils soient aussi de bons « communicateurs » et qu'ils respectent une forme d'éthique dans la communication. Mais comment donner forme à cette éthique et comment communiquer intelligemment une mauvaise nouvelle ? C'est à ces impérieuses questions qu'ont réfléchi Johanne Patenaude, Claudio Lorenzo, Martyne-Isabel Forest, Charles-Henri Rapin et Roger Ladouceur. En exergue de leurs textes, nous aurions pu mettre cette belle phrase de Nietzsche : « Nous cherchons les mots, peut-être cherchons-nous aussi les oreilles» (Nietzsche, 1993, p. 351). En effet, si la parole nous manque, ce n'est pas toujours faute de mots, c'est parfois faute d'oreilles. Les médecins ne sont pas toujours prêts à écouter les réactions de leurs patients à une mauvaise 
nouvelle et les patients ne sont pas toujours prêts à entendre ce que les médecins cherchent à leur dire avec plus ou moins de doigté.

Entre le médecin et le patient, les peurs sont donc nombreuses. Dans son article traitant d'enjeux éthiques reliés aux traitements avec dialyse rénale, Jocelyne SaintArnaud en identifie plusieurs, qui sont d'ailleurs susceptibles de réapparaître dans bien d'autres situations. Pour les praticiens : peur de la mort, peur de constater les limites de la thérapie, peur des poursuites; pour les patients: peur de l'opposition des membres de l'équipe de soin ou des proches, peur de la douleur; chez les proches: peur de la désapprobation sociale, peur de l'abandon du malade par l'équipe de soins, etc.

Heureusement, pour le patient comme pour le médecin, la mort n'est pas toujours l'objet d'une crainte absolue. Dans son film aussi bien que dans son texte, Lina Moreco nous rappelle qu'il arrive que la mort devienne l'ultime objet de désir. Dans de tels cas, il est important que les soins médicaux soient dictés, non par l'appât du gain ou la quête de savoir, mais par l'intérêt du patient. À ce sujet, le testament biologique, bien qu'il soit encore très peu utilisé au Québec, entre autres par les personnes ayant recours à la dialyse rénale (Jocelyne Saint-Arnaud), apparaît aux yeux de Lina Moreco comme un des moyens les plus efficaces pour exercer son droit de maîtrise sur la mort.

\section{TOUTE MORT}

\section{EST-ELLE MEURTRE ?}

Que la mort soit désirée ou crainte, elle s'annonce toujours - ou presque - dans la souffrance. Et qui plus est, cette souffrance est parfois infligée en toute connaissance de cause. Le philosophe Emmanuel Lévinas va même jusqu'à écrire qu' «il faut penser tout ce qu'il y a de meurtre dans la mort » (Lévinas, 1991, p. 81). La mort par lapidation en est un exemple tristement éloquent. C'est ce qu'illustre l'enquête historique que je signe sur la peine de mort par lapidation. Malheureusement, ce mode d'exécution qui remonte à une époque très ancienne n'a pas perdu de son actualité. Certes, nos pays occidentaux ignorent cette pratique, mais les codes pénaux de l'Occident sont encore loin d'être parfaits. On n'a qu'à penser à l'application de la peine de mort aux ÉtatsUnis pour s'en convaincre. Les statistiques données par Mannion (1999, p. 43-50) sont à ce sujet significatives : les États-Unis sont (avec l'Arabie Saoudite, l'Iran, l'Irak et le Yémen) l'un des cinq pays qui, depuis 1990, ont exécuté des prisonniers qui étaient âgés de moins de 18 ans au moment du crime. Ils viennent en tête dans le monde pour l'exécution d'enfants. Depuis 1976, au moins 27 accusés handicapés mentaux ont été exécutés et environ 12 à $20 \%$ des prisonniers dans le couloir de la mort entrent dans cette catégorie. En 1995, sur 3054 prisonniers dans le couloir de la mort, $43 \%$ étaient des Noirs ou appartenaient à des minorités. Force est de constater que si l'application de la Sharía témoigne d'une culture machiste, la peine de mort aux États-Unis, elle, représente la partie émergée de l'iceberg d'un système judiciaire vicié dans une culture montrant de fortes tendances racistes.

Comme la lapidation ou la chaise électrique, la guerre est un autre meurtre orga-

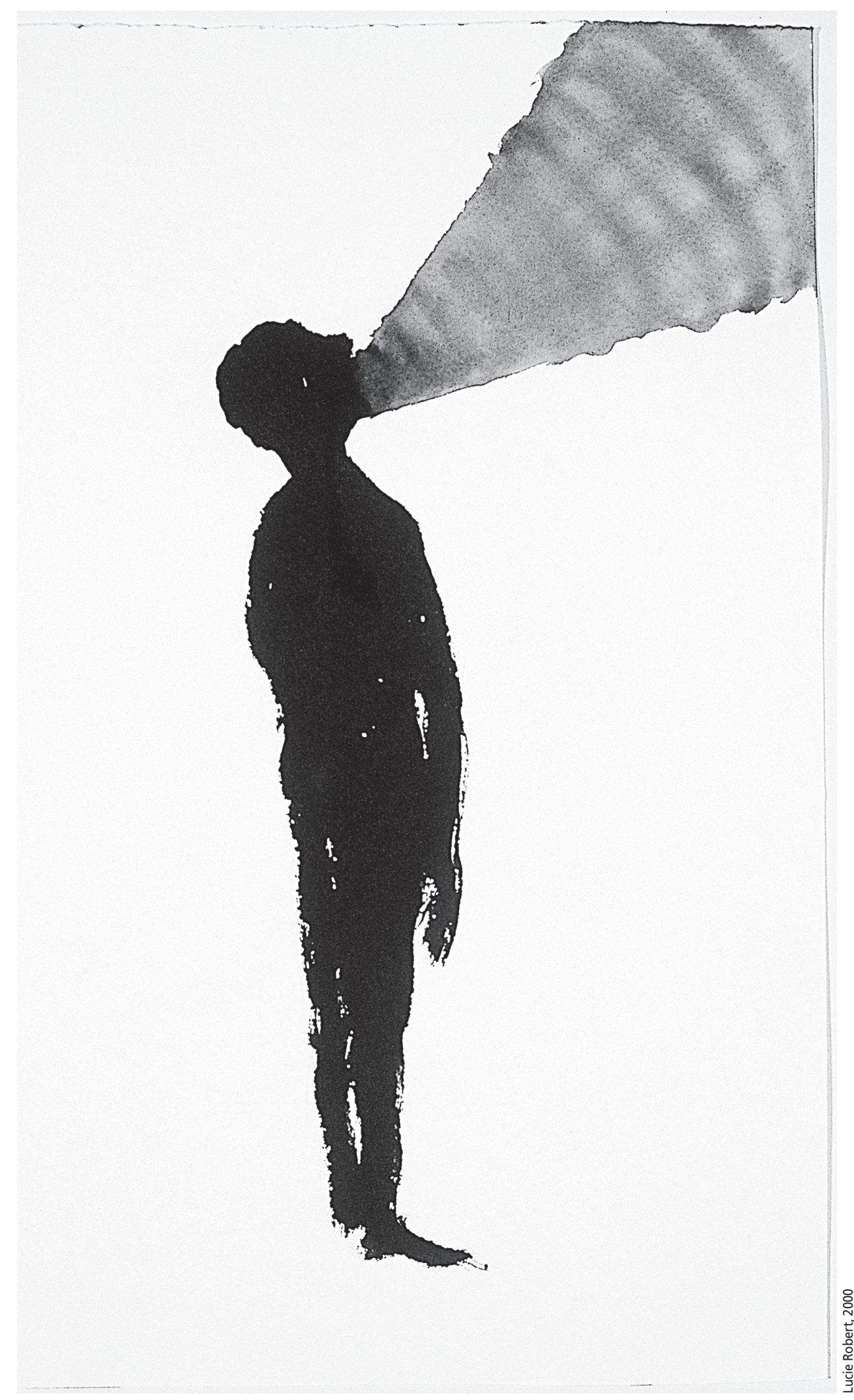


nisé. Pour en parler, la parole a été donnée à un capitaine de l'armée canadienne: Michel Sartori. En montrant jusqu'à quel point le déni de la mort caractérise l'endoctrinement militaire, ce texte laisse voir l'être humain derrière le soldat. Dans ce milieu pourtant sans cesse confronté à la mort, cette dernière ne semble acceptée qu'à « la troisième personne » (Jankélévitch, 1977, p. 23). Pour l'homme armé, la mort est avant tout ce qu'il destine à l'ennemi. Comme l'homme primitif de Freud, le soldat, donc l'être humain, est foncièrement incrédule à l'égard de sa propre mort; par ailleurs, il croit très aisément à celle de ses ennemis, il la désire même, passionnément et sans fausse honte. C'est ainsi qu'à en juger par ses désirs inconscients, l'être humain est comparable à un assassin qui se croit immortel (Freud, 1963, p. 253-267). Ce jugement de la psychanalyse semble particulièrement approprié pour l'armée américaine. Il est vrai que les statistiques alimentent ce phantasme d'une armée invincible. Par exemple, le nombre des victimes militaires dans le conflit qui a opposé l'Irak et les États-Unis est révélateur: environ 100000 soldats irakiens tués, pour 115 soldats américains. Ce «rapport (un pour mille) est unique dans l'histoire militaire du monde.»(Ramonet, 1997, p. 114).

\section{DIRE LE TRAGIQUE}

Bien entendu, le déni de la mort n'est aucunement le monopole des militaires. Selon Luc Ferry, il caractérise même l'attitude des Modernes:

Pour nous, Modernes, la signification de la mort s'est peu à peu obscurcie. Athées ou agnostiques avertis, nous préférerions mourir sur le coup, sans souffrance et, si possible, sans y penser. Toute méditation sur la mort nous semble superflue, peu «virile», et pour tout dire, pathologique.

(Ferry, 1996, p. 12)

Mourir sur le coup, cela peut difficilement fermer la porte au tragique; pensons par exemple au drame que vivent les familles qui perdent un bébé. Pour mieux comprendre l'impact de la brutale séparation que provoque la mort subite du nourrisson, Suzanne Mongeau explore les diverses significations que les mères accordent aux pratiques mises en place par elles, leurs proches et les différents intervenants impliqués. Cette éclairante analyse mériterait de se poursuivre en abordant aussi les difficultés éprouvées par les pères qui ont à faire le deuil de l'enfant perdu.

Paradoxalement, dans son enquête sur les lois juives relatives aux éloges funèbres,
Guylaine Cyr nous apprend que les textes législatifs du monde juif ne permettent pas d'éloge funèbre pour un enfant décédé en bas de trois ou quatre ans, voire de six ou sept ans. Ce fait est d'autant plus étonnant que l'éloge funèbre en tant que parole sur la vie du défunt fait précisément partie du travail de deuil.

À certaines époques de l'histoire, une oraison funèbre pouvait jouer un tout autre rôle que celui de favoriser un travail de deuil individuel : une dimension politique entrait en jeu, à l'échelle de toute une société. Dans son étude de deux des cinq oraisons funèbres destinés au chancelier Michel Le Tellier, deux de ces oraisons ayant été prononcées à plus de dix ans d'intervalle dans une France monarchique, Luc-Normand Tellier explique bien comment ces prises de parole servaient des idéaux différents, en ce qui concerne la vie, la mort et la postérité.

La mort s'annonce non seulement dans les lieux de culte, mais aussi sur... les talons de chèques. Certains chiffres ne font-ils pas référence à la perte inéluctable du statut de travailleur: le départ à la retraite? L'espérance de vie moyenne du salarié n'est-elle pas ainsi quasiment chiffrée? Les conditions de vie des retraités pourront varier selon la rente qu'ils recevront et l'article de Jocelyne Thériault fait bien voir que le critère économique n'est pas le seul en jeu. Aisée ou pas, la retraite peut aussi être vécue comme une mort sociale, comme une véritable perte d'identité.

Le travail du photographe, un peu comme celui du retraité, est aussi un travail de deuil, un travail où la mort et la vie sont inextricablement liées. "Faire des photos, c'est une démarche de deuil, cela peut-être le deuil du lieu, le deuil du moment, le deuil des êtres » (Danziger, 1994, p. 113). Cependant, faire des photos, c'est aussi s'assurer que le passé est désormais aussi sûr que le présent. C'est pourquoi Barthes a également raison lorsqu'il écrit que «la photographie a quelque chose à voir avec la résurrection » (Barthes, 1980, p. 129). C'est à ce lien mystérieux entre la mort et la vie que nous donnent à penser les photos sélectionnées et réalisées par Alain Paiement pour accompagner le lecteur d'un article à l'autre.

Voilà ! La table est servie, discrètement, avec une lumière tamisée et des mots non prononcés. Peut-être certains trouveront-ils qu'il y a un peu trop d'obscurité pour s'attabler, mais il est bien qu'il en soit ainsi, car trop de lumière aveugle. Les idées trop claires ne coupent-elles pas l'appétit de savoir? Au contraire, les idées qui jouent avec l'ombre alimentent la réflexion qui pourrait se poursuivre sans fin.

\section{Bibliographie}

BARTHES, R. (1980). La chambre claire. Note sur la photographie, Paris, Seuil.

DANZIGER, C. (1994). "Clichés. Entretien avec Nicole Godevais», Deuils, Paris, Autrement, p. 112-116.

FERRY, L. (1996). L'homme-Dieu ou le Sens de la vie, Paris, Grasset.

FREUD, S. (1963). Essais de psychanalyse, traduit par S. Jankélévitch, Paris, Payot.

JABÈS, E. (1984). Le livre des marges, Paris, Fata Morgana.

JANKÉLÉVITCH, V. (1977). La mort, Paris, Flammarion.

LÉVINAS, E. (1991). La mort et le temps, Paris, L'Herne.

MANNION, John (1999). «La peine de mort aux États-Unis», Concilium 283, p. 43-50.

NIETZSCHE, F. (1993). Le gai savoir, traduction de H. Albert, Paris, Librairie générale française.

PAZ, O. (1982). O Arco e a Lira, Rio de Janeiro, Nova Fronteira.

RAMONET, I. (1997). Géopolitique du chaos, Paris, Galilée. 